\title{
Iniciación deportiva: la preocupación por el método es una reducción epistemológica
}

Sports initiation: the concern for the method is an epistemological reduction

Germán Hours

Universidad Nacional de La Plata, Argentina

gerhours22@gmail.com

\section{Resumen:}

La Educación Física se ha preocupado y ocupado desde hace mucho tiempo por encontrar las formas más generales de poder intervenir de manera general en las prácticas que aborda, en especial, las deportivas. Con la idea de iniciación deportiva, actualmente convertida en una corriente de enseñanza y una teoría sobre la enseñanza deportiva, todo el debate teórico sobre el deporte quedó reducido a la cuestión metodológica. Desde la perspectiva con la que se configura este artículo, producto además de la investigación científica que lo posibilitó, este reduccionismo anula la posibilidad de la revisión metodológica, en especial porque el mismo es producto de una concepción natural del sujeto, las prácticas y el mundo. En definitiva, la iniciación deportiva y el deporte escolar se ubican dentro de esos cánones evolucionistas preestablecidos, adaptándose a los diferentes ritmos de maduración biológica del ser humano.

Palabras ClaVE: Deporte escolar, Iniciación Deportiva, Método, Naturalismo, Normalización.

\section{Abstract:}

Physical Education has been concerned and occupied for a long time to find the most general ways of being able to intervene in a general way in the practices it deals with, especially sports. With the idea of sports initiation, which has now turned into an approach of teaching and a theory about sports education, all the theoretical debate on sport was reduced to the methodological question. From the perspective which serves as basis for this article, as well as the scientific research that enabled it, this reductionism nullifies the possibility of methodological revision, especially because it is the product of a natural conception of the subject, the practices and the world. In short, sports initiation and school sports are located within these pre-established evolutionary canons, adapting to the different rhythms of biological maturation of the human being.

KEYwORDS: School sport, Sports Initiation Method, Naturalism, Standardization.

\section{INTRODUCCIÓN}

Para comenzar a plantear algunas cuestiones que a continuación se desarrollan, se debe señalar que todos los argumentos presentados aquí, son producto de diversas investigaciones formales ${ }^{1}$, que se han desarrollado de manera articulada durante un tiempo prolongado, estableciendo diversos análisis de una gran cantidad de textos que componen el universo teórico de la Educación Física y la enseñanza deportiva de las últimas cinco décadas, abordajes que a su vez, han permitido diferentes producciones teóricas y trabajos de posgrado. Estas investigaciones permiten definir de manera sintética, que las tendencias actuales que conforman este universo teórico de la Educación Física, le atribuyen al deporte un significado indiscutible en el desarrollo individual y social. Campo conceptual que, de forma general, consideran lo deportivo, y en especial la actitud deportiva, uno de los valores más preciados de la condición humana. Entre estas teorías, se encuentra la Iniciación Deportiva Española [en adelante $I D E$ ], que luego de llevar varias décadas inundando el campo con textos que refieren de manera general y específica a la enseñanza deportiva en la niñez, indudablemente ha logrado el gobierno teórico sobre esta materia. En este sentido, todos los supuestos que la $I D E$ plantea, se apoyan en la idea de que estas prácticas son las que más pueden materializar las posibilidades del niño en su presente y a futuro, reafirmando en cada concepto que es innegable el valor y los alcances que tiene para el desarrollo integral del ser humano. Con argumentos que en su estructura discursiva aparentan cierta cientificidad, estas ideas que reivindican la cuestión más íntima del individuo y la potencialidad social de los deportes, aducen 
construir sus saberes a partir de investigaciones que provienen del campo de la Medicina, en articulación con las teorías de la Psicología del Desarrollo y de cierto progresismo establecido por lo que denominan los avances pedagógicos más actuales. Cuestión que le ha permitido configurarse y autoproclamerse al mismo tiempo, como un posicionamiento casi inobjetable, que posee una de las recetas más eficaces para garantizar la vida plena del individuo. Sin embargo, cuando se profundiza en los análisis de sus contenidos teóricos, las conclusiones que se pueden establecer, determinan que este tipo de postulados se encuentran mucho más cerca de ser una creencia, un dogma o una doctrina ${ }^{2}$, sin dudas, un estatuto político, más quede un saber realmente verificable epistemológicamente, aun cuando se esfuercen por así definirlo.

Una de las características más relevantes de esta forma de pensar lo deportivo, es que establece una revaloración y sobrevaloración de lo metodológico que va más allá del mero análisis operativo que puede suponer, pero que desde esta perspectiva de análisis, muchas veces obstaculiza o anula toda posibilidad epistemológica. En concreto, estas formas de pensar parten de la idea de que tanto el deporte, como su implicancia para el desarrollo individual y social, son incuestionables. En definitiva, al establecer tanta contundencia en sus principios teóricos y en sus fundamentos, no requiere profundizar en ellos, siendo es por esta cuestión, que orienta su preocupación casi exclusivamente hacia las formas de transmisión, es decir, al problema del método. La reducción del debate a la mera discusión metodológica, es una forma de cerrar toda posibilidad de penetración hacia una dimensión del debate que ponga la mirada tanto en lo teórico como en lo político, precisamente por ese valor indiscutido que posee el deporte; lo instrumental pasa de esta manera a ser el eje que estructura vertebralmente todos sus postulados y las orientaciones para la práctica, en especial, las docentes.

\section{LA PREOCUPACIÓN POR EL MÉTODO REFUERZA LA IDEA DE LA NATURALIDAD DEL SER}

La base conceptual principal de estos argumentos se encuentra en la naturalidad que se le asigna al deporte, en relación con la condición humana, que obviamente también es pensada desde su naturalidad. Esa naturalidad que la Educación Física siempre se ha esmerado por asignarle al deporte y a la práctica deportiva -como a todas las prácticas corporales-, reduce las bases teóricas que posibilitan una discusión más profunda, por los mismos supuestos que sirven de fundamento teórico, es decir, por una idea de cuerpo, de individuo, de práctica y hasta de lo disciplinar y de su propio objeto de estudio, que borra toda posibilidad de repensar y generar prácticas y abordajes conceptuales diferentes, en tanto es la propia naturaleza la que define las condiciones de posibilidad. Si el deporte deviene de una cuestión natural, entonces su existencia y su importancia no puede ser cuestionada y finaliza por ser definido como propio y constitutivo de la condición humana. Es entonces que, plantear la discusión exclusivamente desde un plano metodológico u operativo es sólo lo que queda para pulir y mejorar en estas ideas. Sin embargo, y éste el punto central de análisis en este trabajo, como afirma Ricardo Crisorio (2007), “¿quién puede dudar, a estas alturas, que la enseñanza es un ejercicio, abierto u oculto, de relaciones de poder, que el conocimiento y la práctica mismos, definidos y transmitidos por un sistema pedagógico, son formas de poder, que aún los modos de instrucción más radicales son conservadores y están cargados con los valores ideológicos de la estabilidad?” (p.4) Poner la mirada en las cosas más superfluas, como lo es sólo pensar y repensar las formas de aplicación, negando la posibilidad de ampliar el horizonte de análisis, como sugiere Crisorio, es también una forma de ejercer poder y se constituye en una dimensión residual de un posicionamiento no sólo teórico, sino también político y filosófico, que establece al ser humano siempre desde su estatuto biológico.

La ciencia y la política moderna, que concretan la conciencia moderna, han definido al método como la organización racional y bien calculada de los recursos disponibles y de los procedimientos más adecuados para alcanzar un objetivo de la manera más segura. De esta manera, el método se debe comprender como la posibilidad de que se garantice lograr que los alumnos aprendan a aprender más, optimizando las formas de hacerlo. Es la forma más segura de poder alcanzar los objetivos fijados por quienes definen y prescriben 
las posibilidades y las acciones más correctas de comportamiento, reduciendo al máximo las desviaciones perjudiciales en el proceso. Al hablar de método, indefectiblemente se debe hacer referencia a Max Weber (1984) y al concepto de burocracia. Método y burocracia en este modelo tienen una relación directa. Para Weber, la burocracia es una forma de organización humana que se basa en la racionalidad, en la adecuación de los medios a los objetivos pretendidos, con el fin de garantizar la máxima eficiencia en la búsqueda de esos objetivos. Racionalidad que implica, tal como lo afirma la idea de deporte escolar que desarrolla la IDE, la adecuación de los medios a los fines. En concreto, para la $I D E$ los límites de posibilidades están definidos por los pasos para abordar lo enseñable,

Ahora bien, para comprender estos cuestionamientos que aquí se realizan, se debe señalar que, en términos estrictos, la metodología define como al conjunto de mecanismos o procedimientos racionales, empleados para el logro de un objetivo, o serie de objetivos, que en general se encuentran en una investigación científica, pero que también componen otras formas o áreas de conocimientos y, en especial, de intervención. La metodología supone técnicas, procedimientos y soportes que operativizan el diseño y la ejecución de un proceso, permiten su dinámica de manera coherente y, por lo general de manera secuencial, en función de los objetivos fijados. Determina directamente también, la necesidad de poseer un amplio conocimiento sobre el saber a abordar, puesto que es la puesta en marcha para su tratamiento. El método, sin dejar de pertenecer al terreno de lo pedagógico, pasa a ser un factor de implicancia y desempeño de lo didáctico, ambas dimensiones coadyuvando y actuando como dispositivos de conformación y consolidación de cierta postura política que sutilmente se instalará en la conciencia del sujeto. La Didáctica es un área de saberes sumamente relevante en este modelo y así siempre se lo manifiesta en los discursos pedagógicos sobre el deporte escolar que la IDE promueve. Raumar Rodríguez Giménez (2015) considera que es posible realizar una distinción entre lo pedagógico y lo didáctico. Para este autor, lo pedagógico remite "a lo deóntico, a aquello que se plantea como necesario, lo que debe hacerse, que implica lo obligatorio y lo normativo, o bien a la teleológico, aquello que involucra la preocupación por los fines y las acciones a alcanzarlos.” (p. 11) Tomando por su parte a lo didáctico como lo instrumental, lo tecnológico, "que puede reconocerse como la última instancia que organiza metodológicamente una secuencia de contenidos en función de unos supuestos psicológicos de aprendizaje (Behares, 2005)” (ib., p 12) De esta manera, siguiendo a este autor, lo pedagógico debiera ser considerado como la matriz fundante desde la cual se estructura organizadamente ciertos valores de normación y normalización, que establece a su vez las condiciones de posibilidad de lo educativo para la constitución del individuo como tal. En este sentido, no es casual que en los discursos de la Educación Física en general y de la enseñanza deportiva en particular -la $I D E$ en especial, por ser una síntesis de ambos-, los análisis metodológicos prevalecen o están por encima de los análisis epistemológicos de los supuestos que son constitutivos en sus discursos. En todo caso, estos últimos, refieren a una epistemología de la sustancias o de lo natural, nunca un saber neutral; ni la práctica, ni el sujeto, ni mucho menos la concepción del mundo, son analizados, cuanto menos, cuestionados políticamente, instalándose como la verdad absoluta que rige toda manifestación social -piénsese en los enunciados de las llamadas Neurociencias para comprender esta cuestión tan compleja-.

La aplicación de un método de trabajo sistemático, es decir, un orden lógico de actuación que se corresponde con un orden lógico de legitimar las cosas, se puede definir como una didáctica de la legitimación. Así también funciona. Es una forma de defender y potenciar esos aspectos que quieren ser destacados, reproducidos, porque se supone que el saber que los habilita ha sido verificado. Funciona como una verdad pedagógica que es funcional a una verdad política. La preocupación por establecer al tratamiento metodológico como el rector del proceso, es una postura política enunciada desde la apoliticidad del discurso que quiere representar. El método en el tratamiento del deporte escolar anuncia principios particulares de cada una de las situaciones a abordar destacando las estructuras constituyente de las mismas. Pero al mismo tiempo, la revisión metodológica aparenta cierta neutralidad sobre lo que se transmite, permitiendo disimular sus supuestos, puesto que sólo refiere a la operatividad de la práctica. Visto de esta forma, el método posibilita, de 
manera encubierta si se requiere, instalar una idea, establecerla como válida y evitar así cualquier interpelación posible.

Los análisis realizados en la investigación que dio forma a este artículo, han demostrado que estos estudios han centralizado las diferentes formas de enseñanza en las cualidades de la acción motora, unificando criterios, universalizando reglas, generalizando los métodos, y estableciendo parámetros de normalidad y anormalidad para la práctica. En general, la bibliografía que se puede encontrar sobre la enseñanza de los deportes, desde la década del ochenta en adelante, se ubica dentro de un plano exclusivamente metodológico que apuntan al mejoramiento técnico y/o táctico. Se refuerza la idea de que "es conveniente seguir un ordenamiento que responda a la secuencia lógica de aprendizaje [...] con el objetivo de que el proceso pueda llevarse a cabo sin mayores inconvenientes." (Suárez y Mirkin, 2000, s/p) Desde esta perspectiva, el método pasa a tener un rol fundamental en la transmisión y el mismo debe asegurar una serie de pasos ascendentes en cuanto a su complejidad para el logro de los objetivos propuestos. Pasos que estarán determinados por el grado de complejidad que implican para el alumno. Los métodos importan tanto como el criterio del educador, las cuestiones técnicas comienzan a ser tenidas en cuenta para plantearse la trascendencia del para qué de la educación.

De la ejecución técnica motora automatizada basada en la repetición sistemática, preponderante hasta comienzos de la década del ochenta, se dio paso al concepto de inteligencia de juego, la cual requería de procesos de aprendizaje, mediados por una correcta selección de actividades y secuenciados en pasos metodológicos ascendentemente adecuados. Los procesos educativos comenzaron desde ese entonces a ser considerados como una interacción significativa profesor-alumno, en la que el profesor debe ser una fuente de motivación y estímulo permanente. Domingo Blázquez Sánchez (1986) es uno de los autores que más contribuye con estas ideas, al afirmar en su texto La iniciación a los deportes de equipo que, "el objeto de este primer libro es abordar el período que precede a la enseñanza directa de cada uno de los diferentes deportes colectivos (baloncesto, balonmano, fútbol, etc.). Esta fase corresponde al primer eslabón de progresión sistemática de enseñanza que debería acabar con el dominio y la práctica de cada especialidad.”(p. 9) Este autor reconoce la necesidad del establecimiento de pasos secuenciales que faciliten y posibiliten el proceso de enseñanza, afirmando que la renovación conceptual y metodológica debe provenir de los avances en los "movimientos de renovación pedagógica", dado que el campo de la enseñanza deportiva ha mostrado una lenta evolución.

Estas teorías que poseen una visión pedagógica de los deportes, sostienen que en el mundo del deporte infantil, hay rígidos métodos de entrenamiento y ligas con competiciones que no respetan las leyes de la naturaleza ni las capacidades mentales y físicas de los niños. Detrás de estas aparentes críticas a cierta mirada funcionalista y triunfalista que se esgrimen sobre el tratamiento deportivo, se establece una clara reproducción de las mismas, muchas veces maximizando las formas de alcanzar esos objetivos que suponen ser criticados. Esto último puede observarse en cantidad de enunciados que se reproducen en general en cada texto. Por ejemplo, para Blázquez Sánchez el entrenamiento en los niños, es decir, la preparación para el rendimiento, ha ocupado un lugar central a partir de la planificación ordenada y metódica con el objetivo de mejorar los resultados y para este logro se impone una disciplina general y rigurosa. Neil Postman (1995), por su parte, asegura que los infantiles ${ }^{3}$ no están preparados para enfrentarse con ciertas garantías de éxito, ni desde el punto de vista de lo físico o biomecánico, ni desde el punto de vista cognoscitivo. Wein (1995) considera que debería tenerse en cuenta sus características, intereses, expectativas, habilidades y capacidades, lo que se constituye en un principio fundamental para la consideración del niño como centro del proceso de enseñanza, tal como las nuevas tendencias pedagógicas lo demandan. Antonio Orta Cantón, José Pino Ortega e Isabel Moreno Contreras (2000), emiten criterios similares al enunciar que "esto dificulta la introducción de nuevos sistema y medios de entrenamiento. Seguimos empeñados en reducir todo a unidades de medición; por lo tanto el concepto de entrenamiento integrado aún está pendiente en los deportes de equipo.” (p. 5) De este modo sostienen que: 
"Plantear ejercicios simplificados que deben seguir los principios pedagógicos: de lo fácil a lo difícil; de lo simple a lo complejo; de lo conocido a lo desconocido. Siempre debemos comenzar por ejercitaciones sencillas y a medida en que se van asimilando, sumarle dificultades nuevas. Esto le va a facilitar al alumno aprender un contenido liberado de otros movimientos, ej.: si queremos enseñar el contenido de patada de crol, comenzamos con los ejercicios más sencillos como tomados del borde donde el alumno no tenga que preocuparse de la flotación, del movimiento de brazos, etc. y posteriormente vamos incorporando mayores dificultades." (ib., p. 7)

Con respecto a la cita anterior, cabe destacar que su enunciado coincide totalmente con los enunciados que constituyen al positivismo, tal como los estableció en 1861 Herbert Spencer. Del mismo modo que para la Iniciación Deportiva, para el Positivismo, la base de la Pedagogía debía ser la Psicología y, más aún, la Biología. Spencer, sostenía que era necesario establecer primero las bases de la Psicología Racional Científica, para fundar el arte de la Educación o Pedagogía, proponiendo los siguientes principios pedagógicos, que se desprendían de las leyes de la evolución. Obsérvese la siguiente cita:

“1) ir de lo simple a lo complejo; 2) de lo indefinido a lo definido; 3) de lo concreto a lo abstracto; 4) la educación del niño debe concordar, en su modo y orden, con la marcha de la humanidad. El supuesto es que la ontogénesis (desarrollo de un individuo) repite la filogénesis (desarrollo global de la especie), y que la ciencia sigue los mismos pasos para avanzar en el niño que en la historia social; 5) ir de lo empírico a lo racional; 6) estimular el desarrollo espontáneo del niño, diciendo lo menos posible y obligándole a encontrar lo más posible, confiando en la disciplina de la naturaleza; 7) guiarse por los intereses y excitaciones del niño: si un conocimiento es agradable para él, es el indicio más seguro de que vamos por camino correcto. Si esto no surge espontáneamente, debe fomentarse su interés, motivándolo para la experiencia. (Spencer, (1861), 1983, 105-112)" (cf. Dussel y Caruso, 2003, p. 153)

Adhiriendo, aunque no se lo explicite, pero también reproduciendo los principios de este pronunciamiento dogmático, la $I D E$ establece que no se deben saltear etapas fundamentales dentro del proceso de enseñanza. A este punto se lo considera fundamental, ya que se consideran que el no respetarlo, podría generar una ruptura en la asimilación y apropiación de los conocimientos impartidos programáticamente. Al respecto, en cuanto a esta secuenciación en la planificación y aplicación, se considera fundamental que avanzar de una etapa a la siguiente, es el objetivo que deberían plantearse los profesores, a fin que el alumno adquiera todas las vivencias que le permitan no sólo la realización de gestos deportivos específicos, sino también se establezca la posibilidad de la comprensión total del movimiento, lo que algunos autores definen como "entenderlo desde el punto de vista motriz" (Orta Cantón, Pino Ortega y Moreno Contreras, 2000 op. cit., p. 7). Concluyendo en que todas las actividades que enriquezcan el acervo motor favorecerán el dominio acabado de un movimiento, desde un punto de vista que implique una concientización del individuo, que favorecerá todos los aspectos de su integralidad, es decir, la correcta intervención docente sobre lo deportivo, favorecerá el desarrollo no sólo motor, sino además, las dimensiones neurológicas, cognitivas, volitivas, afectivas y sociales.

Sin embargo, y a pesar de los esfuerzos por plantearlo de manera de manera única y fundamental, lo metodológico nunca puede anteceder al saber teórico y político que le permite establecerse, aunque también requiere de un saber específico para configurarse. El método nunca es antes que el tipo de epistemología que lo posibilita, y en su aplicación se pueden rastrear las huellas de esa forma de pensar que le permitió su configuración. En este punto es necesario retomar a Giles (2008), quien del mismo modo cuestiona estas ideas, al proponer replantear científicamente la disciplina, aunque reconociendo que "la discusión con la ciencia es compleja ya que pretendemos hacer ciencia pero abandonado los presupuestos de la ciencia moderna y sus pretensiones de objetividad y generalización" (p. 6) En definitiva, reducir la discusión sólo a lo metodológico es una forma de hacer política que denota una posición, tanto naturalista como dominante de pensar, en tanto sólo se es concebible si se lo reconoce como parte del esfuerzo por mantener el status quo impuesto por la [con]ciencia moderna. Con intereses que corren por fuera de una razón únicamente educativa, la generalización y estandarización prescriptiva que estas ideas difunden, adopta formas que suponen una superación pedagógica, dejando ver que en la preocupación por lo metodológico "la Educación Física [y la enseñanza deportiva] ha estado atravesada por discursos totalitarios, universales y acabados que 
respondieron a la ciencia, el Estado, a Dios y a las corporaciones de profesores" (ib., p. 7), sin pensar realmente en el sujeto y en sus prácticas.

\section{Patrones motores básicos. Supuestos universales}

Un de las bases argumentales que posibilita consolidar este modelo y que a la vez, ha hecho posible reducir la discusión casi exclusivamente a lo metodológico, se basa en la creencia de que existen patrones motores básicos en los individuos, que aparecen de manera natural durante los primeros años de vida y que, mediante determinadas prácticas como la del deporte escolar, pueden ser desarrollados de la manera más conveniente.

Los patrones básicos comunes permiten establecer un orden lógico y metódico, una metodología ascendente en su complejidad que, en consecuencia, prescribirá una dificultad ascendente también en el diseño programático. Así lo piensa por ejemplo Begoña Llorente Pastor (2000), al asegurar que "las habilidades se desarrollan lentamente por etapas, pasando de una forma primitiva, a una forma menos primitiva, a una forma medianamente hábil y, en fin -después de mucha práctica- a una forma muy hábil, de esta manera la observación del adulto debe realizarse sobre la adecuada evolución de los patrones motores básicos." (p. 24) Este planteo, establece que madurar esos patrones básicos de movimiento que conducirán a las mejores formas deportivas no es de inmediato, por lo que, a medida que el niño se enfrente con diversos problemas locomotores que los juegos deportivos y el deporte escolar le presente, paulatinamente se generará un refinamiento de esos patrones y la adquisición de nuevas habilidades motoras. Es así que la diferente combinación de movimientos básicos como correr, saltar, golpear, atrapa, lanzar, etc., generará, a partir de su sistematización, la sincronización necesaria para la aparición de formas superiores o más complejas de movimiento y de habilidad. Descripción que permitió el diseño de una serie de pasos secuenciales que garantizan la eficacia deportiva. Este orden metodológico indica que actividades orientadas hacia el deporte escolar y el tratamiento de peste en concreto, establecido gradualmente desde edades tempranas posibilitará los aprendizajes necesarios para que en su evolución deportiva el niño pueda realizar los ajustes necesarios e imprescindibles para el aceptable rendimiento deportivo final, en concordancia con la evolución de esos patrones básicos comunes que naturalmente contiene el ser bumano. Como se señaló anteriormente, se trata de una graduación y sistematización que sólo puede ser ordenada desde la concepción de lo natural como agente rector, que opera en las posibles estrategias que desde ella se puedan diseñar; es también la posibilidad de establecer un orden de la clase y una diferenciación de roles bien determinada a partir de las posibilidades que el orden natural define. En este enfoque se reafirma la idea de que los patrones de movimiento ya madurados contienen secuencias de movimientos fusionados que parten de la articulación de esos patrones básicos y que permiten la transferencia de movimientos de un deporte a otro. Por ejemplo, Cristian Morales Achig (2015) considera que "el cambio progresivo en el comportamiento a lo largo del ciclo de la vida [...] es un proceso secuencial, relacionado a la edad cronológica, que presenta una evolución desde movimientos desorganizados y poco habilidosos a la ejecución de habilidades motoras altamente complejas” (p. 25). La biología sin dudas es la matriz fundante de esta racionalidad.

El grado de competencia de un individuo frente a un objetivo determinado estará dado por el grado de competencia corporal adquirida por el aprendizaje de movimientos, articulado con la capacidad de generar una respuesta ante múltiples posibilidades de respuestas, que es posible y se ajusta en la relación entre la capacidad individual innata y el ordenamiento con el que se le ha sido enseñado el deporte. Por lo tanto, estas ideas suponen que las experiencias educativas orientadas por el deporte escolar, deben guiar el proceso de maduración hacia una consecución óptima, favoreciendo que se desarrolle al máximo el potencial de cada individuo en cada momento específico establecido por su evolución natural. En este punto es necesario pensar en las fases sensibles para el aprendizaje motor o en los periodos criticos establecidos ambos por el saber psicomédico, que han sido promovidos por el saber pedagógico. De esta manera, se expone su práctica posibilita hacer del individuo alguien válido y funcional, al transmitir y desarrollar una serie 
de conocimientos, instrumentos y habilidades que se consideran básicas dentro de las diferentes formas de agrupar las diversas prácticas deportivas. En este caso, la capacidad de ejecución es contundente y determina la capacidad general del individuo, porque la ejecución implicó con anterioridad un nivel de análisis cognitivo, cuestión que permite reforzar la idea de que lo metodológico supone el saber constituido. Del mismo modo, se sostiene la idea de que su práctica favorece la maduración del ser humano en todas sus dimensiones; la maduración, en consecuencia, proporciona disposiciones y elementos para el desarrollo multidimensional del individuo, dotándolo de capacidades que subyacen de forma innata en él, pero requiriendo del aprendizaje para que se desarrollen con plenitud.

Con la idea de que existen patrones motores básicos, la $I D E$ produce, y reproduce, un conjunto de discursos que afirman que todo parte de considerar que lo específicamente humano en los movimientos del hombre, ha sido heredado biológicamente de sus antepasados; mediante el deporte escolar éstos entran gradualmente en acción y se manifiestan exteriormente, en la medida que el substrato neuromuscular del aparato motor alcanza el nivel de madurez necesario y esperado. En esta idea se ve como las Neurociencias son la base conceptual con las que la ciencia moderna justifica, no sólo el deporte escolar, sino también todas las manifestaciones humanas, obsérvese que las edades deportivas son consecuentes con las edades escolares. Al respecto, y entendiendo que las Neurociencias se constituyen en la síntesis más acabada de la ciencia al servicio del ideal moderno y del capitalismo, y que las teorías de la enseñanza deportiva han promovido sistemáticamente, Nora Merlin (2017) afirma que, "el discurso apolítico de las Neurociencias convierte intereses económicos y empresariales en conocimientos neutros instituidos como verdades." (p. 1) La enseñanza deportiva, al establecerse a partir de todas estas premisas neurocientíficas, a la vez naturalistas, difunde ciertos intereses que son funcionales a un sistema que ha desarrollado de la práctica deportiva una increíble empresa que se mueve al compás de las ganancias que genera, penetrando en el ámbito educativo con un carácter pseudo-científico, como parte del discurso pedagógico y en nombre de lo social del ser humano.

\section{LA PREOCUPACIÓN POR EL MÉTODO ES UNA NORMALIZACIÓN}

Sin dudas, la enseñanza deportiva plasmada en ideas y métodos como el que representa y sintetiza el deporte escolar, es una técnica que establece mecanismos que permiten diseñar, comprobar y clasificar la conducta humana, midiendo las variaciones y los efectos del individuo ante una situación en particular y los grados de normalidad evolutiva, a semejanza de las formas como se aplica en las ciencias naturales y biológicas. Los textos muestran que las ideas que pretenden pensar en lo social conllevan las ideas biológicas como base estructural. Apoyándose al mismo tiempo en disciplinas como la Psicología del Desarrollo, la Pedagogía y la Didáctica fundamentalmente, y estableciendo parámetros de medición y comparación, minimiza la posibilidad de que se reproduzcan desviaciones de los individuos, o de los elementos constitutivos, que componen o forman parte de un grupo social determinado. Con resultados establecidos apriori, puesto que en su programación se encuentran programados también los resultados esperados, y encontrando formas para distintos niveles de adaptabilidad, establece los parámetros de comparación que permitirán analizar, corregir y encauzar los casos conflictivos para la sociedad, por los cánones aceptables de comportamiento, impuestos y predeterminados. No obstante sus postulados liberadores y potenciadores del desarrollo y la posibilidad de expresión del individuo, permite separar las partes del todo para conseguir analizar el todo por separado y lograr un conocimiento y un control más detallado de cada parte y de las relaciones que existen entre unas y otras, a fin de explicar las relaciones que a simple vista no aparecen solucionables predeterminadamente. Es decir, permite reunir bajo criterios racionales elementos que pueden hallarse dispersos o en tensión para crear una nueva totalidad, una nueva realidad. En síntesis, en el método que implica la iniciación deportiva, se organizan y estructuran las técnicas concretas -el deporte escolar, por ejemplo- que servirán para conseguir un objetivo determinado, es decir, determinada formación del individuo. 
El método por el cual el niño puede acceder a la práctica deportiva específica en una etapa posterior, y en el que el deporte escolar se constituye en un eslabón imprescindible para esta forma de pensar. Implica una sistematización y ordenamiento de las prácticas sociales, que constituye un principio general ordenador, que a través de la correcta aplicación tendrá alcances hacia lo particular, permitiendo aceptar como evidencia sólo lo que es evidente a la comprensión, descomponiendo al individuo, en tanto objeto de tratamiento, en un problema que se resuelve a partir de analizar y discernir sobre sus elementos constitutivos. Del mismo modo que las reglas del Positivismo, el deporte escolar es presentado como parte de un procedimiento, que formula conclusiones por deducción, que a su vez se confronta con la realidad, como medio para ver si estas conclusiones se condicen o entran en tensión con ella, y, mediante este análisis, prestar atención si éstas definitivamente deben ser reafirmadas o abortadas. Tomando como referencia a Valeria Emiliozzi (2011), se puede afirmar que el deporte escolar, en esa supuesta apertura didáctica que varios autores reivindican, "sienta sus bases en un educación humanista, no hace otra cosa que implementar un modelo educativo que busca desarrollar un deber ser, un efecto normalizador sobre el sujeto.” (p. 26) En consecuencia, el deporte escolar puede ser pensado como una forma de normalizar y la supuesta autonomía que éste lograría, como una norma y no como un fin. Obsérvese entonces lo que Emiliozzi afirma con respecto a la normalización, al señalar que "el concepto de normalización juega un papel fundamental, en tanto que ese proceso de regulación de la vida de los individuos y de las poblaciones se ejerce bajo el dominio de la norma. La norma es lo que puede aplicarse tanto a un cuerpo al que se quiere disciplinar como a una población a la que se pretende regularizar" (ib., p. 228 y 229$)$.

\section{REFLEXIONES FINALES}

Determinar un método único y sistematizarlo, trasladándolo a todos, a todas las categorías, y a diferentes disciplinas, como la IDE siempre pretendió -recordar la idea de Blázquez Sánchez (1986opcit) de encontrar una forma universal, un manual de enseñanza que sirva para todos los deportes-, se relaciona más con una política que persigue intereses particulares, más que con las variables que se enuncian como intervinientes en la enseñanza de los mismos. Se trata de una normalización evidente, que por otra parte, la IDE no niega, sino que justifica explícitamente. Como lo ha señalado Georges Canguilhem (1980) esto ha establecido una explicación teórica basada en general por un determinismo con sustento único en la estructura y la configuración del cerebro, y en sus funciones intelectuales, que ha vuelto tan ambigua como burda, sus razones como sus efectos.

Al mismo tiempo, instalar la preocupación sobre el método en materia de enseñanza, implica un reduccionismo que no puede disimularse y posiciona la práctica desde un lugar tan natural como previsible, precisamente porque esa es la idea. Repleto de supuestos que contienen resultados obvios, sin tomar en consideración las particularidades, tanto de los sujetos que intervienen en las prácticas corporales, como de las prácticas mismas, entendidas éstas como las posibilidades de manifestación que tiene el sujeto, siempre sin saberlo, define los parámetros que condicionarán su vida. Pero esta preocupación por el método, es más complejo aun, es una forma de despolitizar una manifestación cultural que se encuentra cruzada por diversas tensiones e ideologías que la configuran, que lejos están de estar determinadas por una sentencia que proviene del orden de lo natural. En este caso, al naturalizar las cosas, se las desprende de lo político y ello es sumamente provechoso para el interés político que conlleva estas ideas: dominar la disciplina, y legitimarse y perdurar institucionalmente, cuestión que también Blázquez Sánchez explicitó claramente en su primer libro.

Una de las formas que más ha colaborado en establecer este tipo de pensar, que ha monopolizado el campo de las prácticas de educación física ${ }^{4}$, estuvo comprendido por el amparo que se encontró en refundar la teoría del aprendizaje a partir de marcos pedagógicos supuestamente más progresistas, pero que, por contrario a lo que insinúan o pretenden definir, responde a viejas costumbres que no hacen más que revestirla de la 
tradicional naturalidad que la humanidad ha aceptado para todo aquello relacionado al sujeto y a su propia definición.

Estas ideas que suelen definirse como modelos, se presentan siempre como propuestas didácticas completas y hasta infalibles, siempre que lo normal y la normalidad se establezcan como agentes reguladores de su aplicación. Estructurada de manera que la teoría y la práctica son dos cosas distintas pero se encuentran siempre relacionadas, aunque la segunda sujeta a las razones que la primera establezca; la práctica es siempre una aplicación posible de una teoría que la antecede. En el caso del deporte escolar, método y experiencia se dan la mano para esbozar los primeros lineamientos de un mecanismo que enmascara detrás de la ciencia moderna, sus deseos de gobierno del campo deportivo en la niñez. Elige un orden de trabajo que se sistematiza por fases ${ }^{5}$, reflexionando que a cada fase le precede y le continúa otra fase que se ordenan de manera lógica desde lo evolutivo. Como este ordenamiento ha de ser transmitido al alumno, bajo esta perspectiva se define que se contribuye al desarrollo de autoaprendizaje del alumno y al mismo tiempo a lograr un mayor interés y confianza del alumno en la materia, cuando en realidad, y así también se lo ha expresado en repetidas oportunidades, se está encausando las conductas individuales y colectivas.

Deporte escolar y desarrollo motor pasan a ser lo mismo. El desarrollo motor y el deporte, al ser considerados como capacidades naturales del individuo, actúan como verdades incuestionables, por lo que, el análisis del método pasa a ser el objeto a analizar. Desde esta perspectiva entonces, la idea de encontrar un método universal, posibilitó el establecimiento del deporte escolar como herramienta metodológica que permita alcanzar los objetivos propuestos, desde una visión evolucionista del individuo. En este mandato instituido e instituyente, el individuo, el cuerpo y sus manifestaciones, quedan reducidas a su más íntima existencia natural, posibilitando un conjunto de estrategias de intervención sobre éstos, que constituyéndose a partir de esa matriz humanista superior, somete del sujeto a las leyes de la naturaleza, transformándolo en un individuo apolítico, universal y abstracto, capaz de ser formateado con un proyecto superior. En definitiva, la preocupación por el método, responde a la razón moderna, que requiere eliminar todo lo bárbaro que hay en el sujeto, para civilizarlo y conducirlo de manera particular y colectiva, evitando al máximo desviaciones que puedan perturbar la norma impuesta.

\section{REFERENCIAS}

Blázquez Sánchez, D. (1986). Iniciación a los deportes de equipo; Madrid; ed. Martínez Roca.

Canguilhem, G. (diciembre de 1980). El cerebro y el pensamiento. Conferencia presentada en Mla Sorbone para el M.U.R.S. Primera publicación en Prospective et Santé, $n^{\circ} 14$. Traducción al español: Ernesto Hernández B., Abril de 2004. Recuperado de: https://es.scribd.com/document/142121481/SEM09-CanguilhemElCerebroyelPensamiento

Crisorio, R. (Septiembre, 2007). La enseñanza como herramienta en la divulgación y apropiación del Conocimiento. Trabajo presentado como ponencia em 'Divulgação e Apropriação do Conhecimento Científico: Intervenção, Agentes e Instituições da Educação Física'. XV CONBRACE/II CONICE. Recife, Brasil. Recuperado de: http://revista.cbce.org.br/index.php/RBCE/article/download/197/204

Dussel, I. y Caruso, M. (2003). La invención en el aula. Una genealogía de las formas de enseñar. Buenos Aires, Ediciones Santillana.

Emiliozzi, V. (2011). Fragmentos de cuerpos despojados: Una arqueología y una genealogía de los discursos del cuerpo en el Diseño Curricular de Educación Física (Tesis de Maestría).Universidad Nacional de La Plata. Facultad de Humanidades y Ciencias de la Educación. Recuperado de http://www.memoria.fahce.unlp.edu.ar/tesis/te.440/ te.440.pdf

Giles, M. (2008). Educación corporal: Algunos problemas [En línea]. Jornadas de Cuerpo y Cultura de la UNLP, 15 al 17 de mayo de 2008, La Plata. Recuperado de: http://www.fuentesmemoria.fahce.unlp.edu.ar/trab_eventos/ ev.691/ev.691.pdf 
Hours, G. (2014).Los discursos de la enseñanza deportiva. Mitos, tradiciones y naturalizaciones. Análisis del discurso de la Iniciación Deportiva española (Tesis de Maestría). Facultad de Humanidades y Ciencias de la Educación, Universidad Nacional de La Plata, Recuperado de: http://hdl.handle.net/10915/34673

Llorente Pastor, B. (10 de octubre, 2000).Pedagogía del entrenamiento deportivo adaptado a escolares. Educación en valores. Jornadas de Deporte Escolar. Bilbao. Recuperado de: http://www.bizkaia.eus/Kultura/kirolak/pdf/ ca_PedagogiaEducacionValores.pdf?hash $=03005$ be31a4774bf1 140cc37fab24641\&idioma $=$ CA

Merlin, N. (2017). Colonización de la subjetividad: las neurociencias [Exclusivo en línea] Revista de Cultura y Política.; Buenos Aires, 15 de marzo de 2017. Recuperado de: http://consultoriobosch.com.ar/colonizacion-dela-subjetividad-las-neurociencias/

Morales Achig, C. (2015). La actividad fisica y su incidencia en las destrezas motrices basicas en los niños de entre 8 y 10 años de edad, de la parroquia de conocoto, canton quito por medio de un campamento vacacional en el periodo 2015. Universidad de las Fuerzas Armadas, Ecuador. Recuperado de: https://repositorio.espe.edu.ec/ bitstream/21000/11481/3/T-ESPE-049283.pdf

Orta Cantón, A.; Pino Ortega, J. y Moreno Contreras, I. (2000). Propuesta de un método de entrenamiento universal para deportes de equipo basado en el análisis observacional de la competición [Exclusivo en línea] Revista EFDeportes, Buenos Aires, año 5, N²7. Noviembre del 2000. Recuperado de: http://www.efdeportes.com/ efd27a/de1.htm

Postman, N. (1995). The End of Education: Redefining the Value of School. New York, Vintage Books.

Rodríguez Giménez, R. (2015). La vida en la encrucijada de lo humano (o lo pedagógico y el hombre en tanto que "vive"). Dossier "Gubernamentalidad y educación". Pedagógica (Montevideo), No 3, marzo 2015 - ISSN: 1688-8146. Recuperado de: http://www.academia.edu/34035169/ La_vida_en_la_encrucijada_de_lo_humano

Suárez, R. y Mirkin, A. (2000). Características del proceso metodológico en natación [Exclusivo en línea]. EFDeportes, Buenos Aires, año 5, № 25. Setiembre de 2000. Recuperado de: http://www.efdeportes.com/ efd $25 \mathrm{a} / \operatorname{metod} 1 . h \mathrm{tm}$

Weber, M. (1964).¿Quées la burocracia?. Buenos Aires, Tauro Ediciones.

Wein, H. (1995).La clave del éxito en el hockey: un óptimo modelo para desarrollar la capacidad de juego. Buenos Aires, Ediciones Preescolar.

\section{Notas}

1 Entre estas investigaciones, se pueden señalar en especial: el proyecto I+D dirigido por Marcelo Giles Las prácticas de enseñanza del deporte: del alto rendimiento a la formación, el camino inverso, código H419, llevado a cabo entre el 2006 y 2009; el proyecto I+D dirigido por Marcelo Giles Los discursos de las prácticas corporales, código H564, desarrollado entre 2010 y 2011 ; el proyecto I+D dirigido por Marcelo Giles Los discursos de la enseñanza de las prácticas corporales, código H616, desarrollado entre los años 2012 y 2015; el PPID que se encuentra en desarrollo, dirigido por Germán Hours Los discursos de la enseñanza de los deportes desde la perspectiva de la Educación Física. Análisis sobre la construcción teórica en el campo desde el comienzo de la Globalización, código PPID/H047;todos ellos pertenecientes al Plan de Incentivos a la Investigación de la Nación, radicados en la FaHCE, UNLP; y la investigación que posibilitó la tesis de posgrado Los discursos de la enseñanza deportiva: Mitos, tradiciones y naturalizaciones. Análisis del discurso de la Iniciación Deportiva española, correspondiente a la Maestría en Deporte, de la FaHCE, UNLP, presentada por Germán Hours en el año 2014.

2 "La Iniciación Deportiva se conforma desde un saber, que pretende ser científico, en el estudio de las estructuras biológicas del movimiento y en el análisis estructural de las diferentes prácticas, pero no logra lo mismo en el análisis político de la práctica deportiva, ni de los sujetos que en ella se manifiestan. Se trata más de un saber construido a partir de la idea del sentido común que la ciencia moderna ha establecido, entendiendo esta categoría como el común denominador de los conocimientos, valoraciones y costumbres, propios de una sociedad determinada. Si se lo piensa de esta manera, el sentido común que la Iniciación Deportiva ha consolidado se convirtió en un saber doctrinario. En este sentido, Foucault (1996) es muy crítico al realizar un agudo cuestionamiento a la doctrina y al saber doctrinario, del cual considera que de no ser porque no posee validez científica, sino que se conforma a partir de la simple aceptación y 
suscripción de un grupo, su estructura se asemeja al saber científico, pero en rigor de verdad, nada tiene de éste." (Hours, 2014, p. 102)

3 Categoría utilizada por el autor.

4 Las minúsculas diferencian la práctica de la disciplina

5 El término fase es muy utilizado en el campo de la enseñanza deportiva. Detrás de esta denominación subyace la idea de un proceso de enseñanza que se configura como una especie de ensamblaje de cada uno de los componentes que comprende son parte de una especie de circuito con el que se piensa al proceso de enseñanza. Es la forma de establecer una diferenciación de cada uno de los componentes del proceso, para establecer de esta manera la relevancia y el tratamiento en particular que sobre cada uno de ellos se puede tener. Esta programación se corresponde con una concepción que debe respetar el orden natural de las cosas y la evolución psico-física del individuo. Entre las formas más utilizadas que se pueden encontrar para ejemplificar esta lógica, se pueden nombrar: las fases sensibles del aprendizaje motor, las fases o períodos evolutivos, o hasta las fases del juego y las fases del entrenamiento, entre muchas otras. Esta denominación se condice con una forma de programar, no sólo el proceso de enseñanza, sino al individuo con el que se va a trabajar, para optimizar sus posibilidades de aprendizaje, a fin a un proyecto deportivo preestablecido, es decir mediante esta especie de ensamblaje, se garantiza la eficacia del proceso, dado que esta mirada supone mayor eficacia en la selección, en el abordaje y en la evaluación de las estrategias que se planteen para la enseñanza deportiva. Si bien el campo deportivo adoptó esta idea de manera concluyente, no es relativa sólo a él, por el contrario, es parte de una forma con la que en general se ha pensado la enseñanza durante toda la Modernidad. 